\title{
Uniportal video-assisted thoracic surgery for a tuberculous collar-button abscess of the chest wall involving ribs: a case report
}

\author{
Hongliang Bian ${ }^{1 \#}$, Baihan Su ${ }^{2 \#}$, Chao Wu ${ }^{1}$, Tianshuo Jiang ${ }^{1}$, Xin Zhao ${ }^{1}$, Fangchao Liu ${ }^{1}$, Keli Lin ${ }^{1}$, \\ Qingshan Gao ${ }^{1}$, Yilei Zhang ${ }^{1}$, Michel Gonzalez ${ }^{3}$, Marco Sperandeo ${ }^{4}$, Kyoji Hirai ${ }^{5}$, Zhiyong Su ${ }^{1}$ \\ ${ }^{1}$ Department of Thoracic Surgery, Affiliated Hospital of Chifeng University \& Institute of Thoracic Trauma and Tumor of Chifeng University, \\ Chifeng, China; ${ }^{2}$ Department of Otolaryngology-Head and Neck Surgery, Department of Otolaryngology-Head and Neck Surgery, Beijing Anzhen \\ Hospital, Capital Medical University, Beijing, China; ${ }^{3}$ Service of Thoracic Surgery, Lausanne University Hospital (CHUV), Lausanne, Switzerland; \\ ${ }^{4}$ Unit of Interventional and Diagnostic Ultrasound in Internal Medicine, Department of Medical Sciences, IRCCS "Casa Sollievo della Sofferenza", \\ San Giovanni Rotondo, Italy; ${ }^{5}$ Division of Thoracic Surgery, Nippon Medical School Chiba Hokusoh Hospital, Chiba, Japan \\ \#These authors contributed equally to this work. \\ Correspondence to: Zhiyong Su. Department of Thoracic Surgery, Chifeng University Affiliated Hospital, Songshan District, Chifeng 024001, China. \\ Email: suzhiyong1967@126.com.
}

Submitted Feb 26, 2020. Accepted for publication Jan 14, 2021.

doi: 10.21037/jtd-20-3504

View this article at: http://dx.doi.org/10.21037/jtd-20-3504

\section{Introduction}

Chest wall tuberculos.is is a common chest wall disease of the ribs, sternum, and chest wall soft tissue secondary to tuberculosis of the lung or pleura. Rib tuberculosis accompanied by collar-button tuberculous abscesses is a manifestation of chest wall tuberculosis. Typically, the lesion penetrates the intercostal space to form abscesses inside and outside the ribs, a superficial abscess under the skin, and a deep collar-button-shaped abscess behind the ribs (1). Chest wall tuberculosis is usually treated with anti-tuberculosis agents and drainage, and the primary surgical treatment is to remove the damaged ribs surgically while clearing the abscess, depending on the specific conditions of the patients $(2,3)$. These operations require large surgical incisions and may also cause extensive chest wall defects, which may leave large scars despite repair with flap transplantation $(4,5)$. Therefore, a surgical method with definite treatment effectiveness and a smaller and more beautiful incision is urgently needed. We have designed and applied a new surgical method-Uniportal-VATS-to remove the damaged ribs and clear away the collar-button abscess in the chest wall by using the incision protective sleeve to set up a tunnel. This method also enables thorough debridement with smaller surgical trauma, which causes the chest wall to be better-looking after the surgery. The outcome was satisfactory during the 6-month follow-up period.

We present the following article in accordance with the CARE reporting checklist (available at http://dx.doi. org/10.21037/jtd-20-3504).

\section{Case presentation}

All procedures performed in studies involving human participants were in accordance with the ethical standards of the institutional and/or national research committee(s) and with the Helsinki Declaration (as revised in 2013). Ethics Committee approval was obtained from the Institutional Ethics Committee of Affiliated Hospital of Chifeng University to the commencement of the study (No. FSYY202002). Informed consent was obtained from the patients.

A 23-year-old male patient was admitted because of an eminence that appeared at the right costal arch, which was found one month ago. The patient had no relevant medical history before. The mass at the right costal arch was sized about $6 \mathrm{~cm} \times 5 \mathrm{~cm} \times 1.5 \mathrm{~cm}$, and it traveled along with the costal cartilage. It had an intact skin surface, where there was no redness, swelling, or ulceration. The skin was soft, with normal temperature. The mass had a sense 


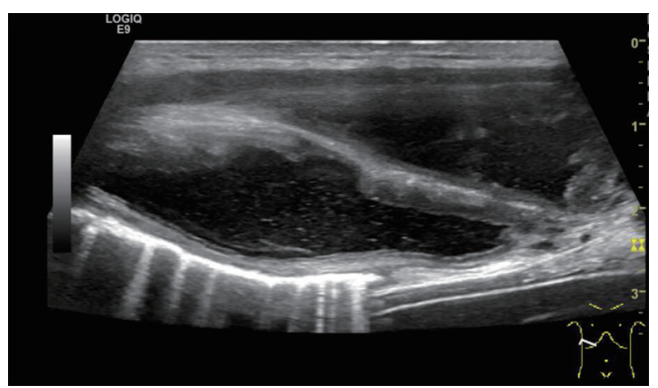

Figure 1 Ultrasound of tuberculous collar-button abscesses of the chest wall.

of fluctuation and blurred border but without tenderness. Auxiliary tests after admission showed weakly positive TB antibodies: TB-specific antigen A: 20; and TB-specific antigen B: 15. T-SPOT.TB was positive (+). Ultrasound showed an multi-cavity anechoic lesion, the pus cavities outside ribcage sized about $7.0 \mathrm{~cm} \times 2.4 \mathrm{~cm}$, and the pus cavities inside ribcage sized about $9.0 \mathrm{~cm} \times 3.0 \mathrm{~cm}$ surround the 7 th and 8 th ribs at the right costal region. The transmission of the sound beam was reduced, and the intercostal soft tissues were visible, although densely dotted echoes were observed. The fluid flowed cross the rupture in the intercostal tissues could be observed after the probe was pressurized. The possibility of a tuberculous collar-button abscess of the chest wall was considered (Figure 1). CT scan revealed an irregular focal hypodense lesion subcutaneously on the right side of the 7 th and eighth anterior periosteal area. The mean CT value was about $14 \mathrm{HU}$ on the plain scan. No enhancement was observed in the hypodense area during the contrast-enhanced scan, although the border of the lesion was enhanced. A diagnosis of the abscess was considered. However, no visible lesion was observed in the lungs (Figure 2A,B).

Diagnostic fine-needle aspiration of the body surface mass revealed pathological necrosis, lymphocyte infiltration, and caseous necrosis. Anti-TB agents were given for three weeks after admission. His liver and kidney functions were normal. However, the chest wall abscess was not relieved after the conservative treatment, and it might be ruptured without surgical intervention.

\section{Preoperative assessment}

Uni-VATS was then performed to aid the removal of the chest wall abscess and the partial rib resection. Repeated tests of sputum specimens showed negative results. There was no toxic symptom of TB infection. His erythrocyte sedimentation rate (ESR) and C-reactive protein level were not elevated. Also, there was no evidence of active pulmonary tuberculosis. Thus, surgical resection of the lesion was possible. The patient's general condition and cardiopulmonary function were excellent and could tolerate the surgery. Other examinations and tests showed no clear contraindications to surgery. Thus, the surgery was performed after obtaining informed consent from the patients.

\section{Anesthesia and body position}

The patient was placed in a supine position, with the right chest raised by $30^{\circ}$ and the right arm abducted by $90^{\circ}$, to expose the right-sided chest wall abscess and facilitate the surgical operation fully. General anesthesia with double-lumen endotracheal intubation was applied. If intrapulmonary lesions were found during intraoperative exploration, they would be concurrently resected with unilateral ventilation of the left lung.

\section{Surgical process}

A $3-\mathrm{cm}$ incision was created at the standard tissue $1 \mathrm{~cm}$ under the abscess. After the subcutaneous tissue and various layers of the muscle were cut open, the abscess cavity was entered (scope: about $5 \mathrm{~cm} \times 5 \mathrm{~cm} \times 3 \mathrm{~cm}$ ). The extra-costal pus cavities were cleared away as possible, and then the incision protector was inserted to set up a tunnel (Figure $3 A$ ). A thoracoscope was placed for exploration. The caseous necrotic fluids in the purulent cavities were cleared away and rinsed with iodophor. It was found that the sinus tract had penetrated the intercostal muscles, where phagocytic destruction of the 7 th and 8 th ribs and the costal cartilage was found. The damaged periosteum of the ribs was peeled off. The 7 th and 8 th ribs were resected by about $3 \mathrm{~cm}$ by using a wire saw (Figure 3B,C). The intercostal muscle bundle (Figure $3 D$ ) was retained, and an abscess cavity sized about $10 \mathrm{~cm} \times 10 \mathrm{~cm} \times 3 \mathrm{~cm}$ outside the pleura behind the ribs were further exposed, where the formation of granulation tissue and fiberboard was visible. After the abscess cavity behind the ribs was cleared away (Figure $3 E$ ), the granulation tissue on the wall of the abscess cavity was removed. Exploration of the deep side of the abscess cavity showed the parietal pleura was intact. After strict rinsing and disinfection with iodophor, streptomycin powder $1 \mathrm{~g}$ was introduced, and a negative-pressure drainage tube was placed (Figure $3 F$ ). Then, the incision was sutured. Compression bandaging was 

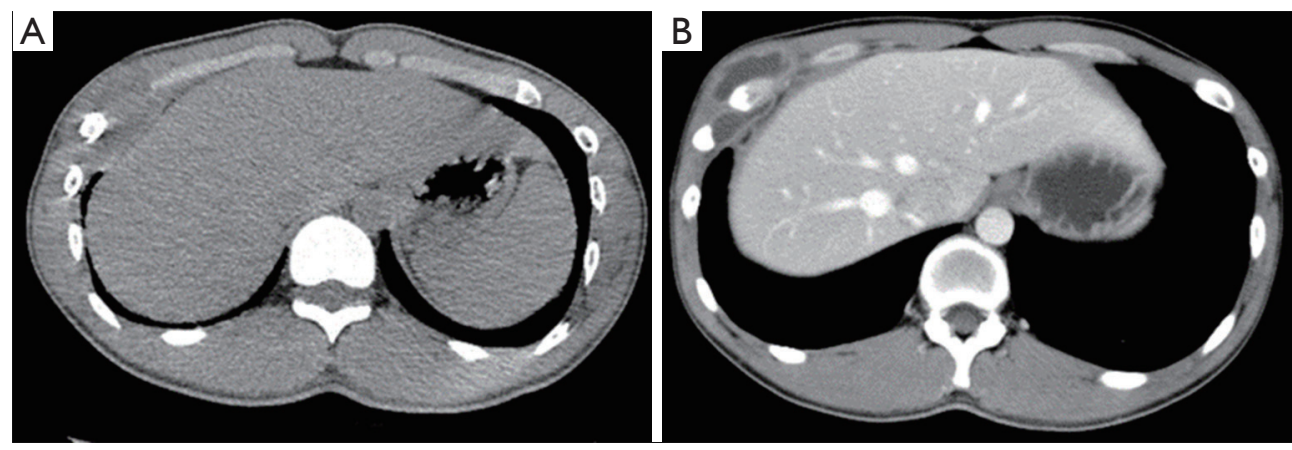

Figure 2 Chest and upper abdominal Computer Tomography (CT) scans reveal an irregular focal hypodense lesion subcutaneously on the right side of the 7th and 8th anterior periosteal area. (A) The mean CT value was about 14 HU on the plain scan; (B) no enhancement was seen in the hypodense area during the contrast-enhanced scan, although the border of the lesion was enhanced. A diagnosis of the abscess was considered.
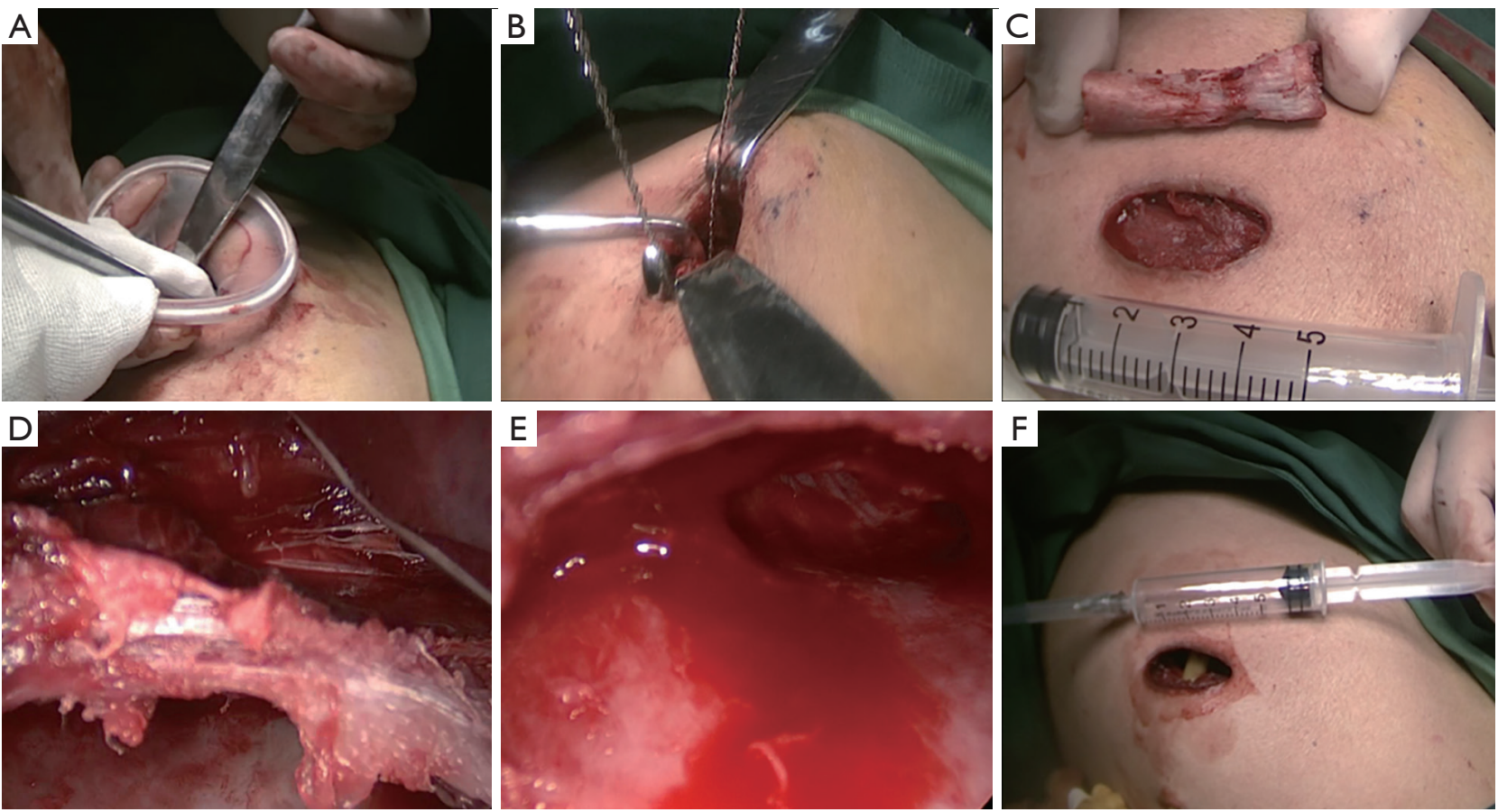

Figure 3 Operative procedure: (A) built a tunnel; (B) remove the damaged ribs with a wire saw; (C) the removed rib segment; (D) the remaining intercostal muscle bundle; (E) thoroughly clean the abscess cavity; (F) place the drainage tube.

applied locally. The postoperative pathology (HE staining). Local tissue necrosis of the 6th and 7th ribs accompanied by multinucleated cell infiltration, consistent with chronic osteomyelitis on the right side. Scale bar, $100 \mu \mathrm{m}$ (Figure 4).

\section{Postoperative management}

The anti-TB treatment continued. Liver and kidney function tests and blood tests were performed routinely. Also, streptomycin $0.75 \mathrm{~g}$ in PD was administered for two consecutive months. Treatment with rifampicin $450 \mathrm{mg}$, ethambutol $500 \mathrm{mg}$, and isoniazid $300 \mathrm{mg}$ continued, along with the continuous use of pyrazinamide $500 \mathrm{mg}$ and liverprotective drugs. The dressing was changed continuously, and the subcutaneous negative-pressure drainage tube was gradually withdrawn. After the incision was well healed, 


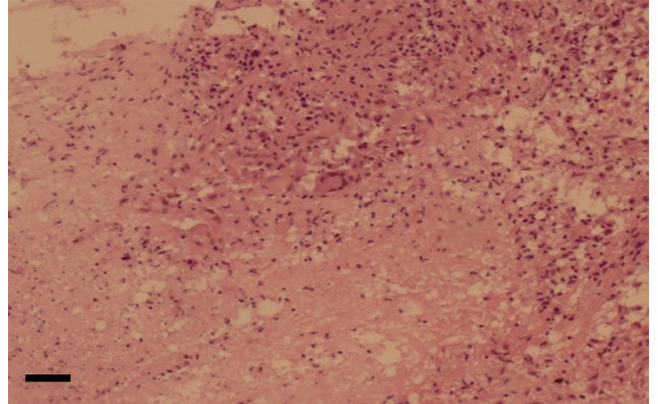

Figure 4 The postoperative pathology (HE staining). Local tissue necrosis of the 6th and 7 th ribs accompanied by multinucleated cell infiltration, consistent with chronic osteomyelitis. Scale bar, $100 \mu \mathrm{m}$.

the patient was discharged. The patient was instructed to continue taking oral anti-TB drugs for one year after discharge and to receive regular live/ kidney function tests and chest radiography. Chest CT performed six months after the operation showed a good recovery (Figures $5 A, B, 6$ ). The patient was satisfied with the treatment result

\section{iMDT discussion}

In our current case, the unmarried young patient has a high cosmetic requirement on his body appearance. Traditionally the disease is treated with open procedures that are associated with a high recurrence rate, and reoperation often results in larger scars. Then, is it possible to design a minimally invasive surgery to remove the deep and shallow abscesses altogether and, meanwhile, resect ribs that have already damaged by tuberculosis? When is the correct timing for such a surgery? How to reduce the recurrence rate? How to select the correct incision? What are the indications for minimally invasive surgery? What will be our treatment plan?

\section{How does a traditional open surgery for chest wall tuberculosis ensure complete removal of abscesses and damaged ribs?}

\section{Inputs from thoracic surgeons Dr. Hongliang Bian}

The key principle of traditional surgery for chest wall $\mathrm{TB}$ is to clear away the pus cavities inside and outside the chest wall entirely and remove the damaged ribs. The healthy ribs may also be resected to make the muscle entirely collapse and avoid unhealed incision or recurrent lesion $(6,7)$. The leading causes of lesion recurrence or unhealed incision include incomplete removal of the abscess, failure to resecting the damaged lesions on the ribs, and insufficient anti-TB treatment. Kim et al. concluded that complete abscess removal (including the resection of the affected chest wall) could reduce surgical risk and reduce recurrence (8). They also recommend the complete removal of abscesses (including resection of necrotic ribs and cartilage) because the abscess may also affect the deep tissues. However, the final decision is usually with intraoperative findings.

According to our experience, chest wall abscess often occurs in young and middle-aged people. We don't have enough evidence to support that the VATS was suitable for elderly people. But in our opinion, if the elderly patient with good physical condition could withstand the surgery strike and medical therapy post-operation, and the lesions could be eliminated completely during Uni-VATS, UniVATS also could be as one of the surgical methods took into account.

\section{Dr. Tianshuo Fian}

(I) If there is a sinus tract in the skin, the skin at the sinus ostium is removed by spindle-shaped resection, and then the pus is thoroughly absorbed, and the diseased tissue in the pus cavity that is connected with the sinus tract is scraped away. After the bleeding is stopped by filling the sinus tract with dry gauze and by electrocoagulation, the traveling direction of the sinus tract is identified to decide the direction and length of the incision to be extended. If the sinus tract is long and the distance between the primary lesion and the sinus ostium is also long, a new incision may be created at the blind end of the sinus tract and along the rib under the guidance of the intra-sinus probe, to expose and remove the primary lesion.

(II) If there is no sinus tract in the skin, create a $3-4-\mathrm{cm}$ incision at the low point of the chest wall, abscess along the running direction of the rib, and make a corresponding arc extension according to the findings of the exploration. If the abscess has penetrated the myometrium and reached the subcutaneous tissue, the pus is thoroughly absorbed, and then the muscle fibers are separated along the sinus tract to reach the abscess. If the abscess has not penetrated the myometrium, it should be separated or divided until the abscess is cut open. After the pus is thoroughly absorbed, quickly scrapes the wall of the abscess. 

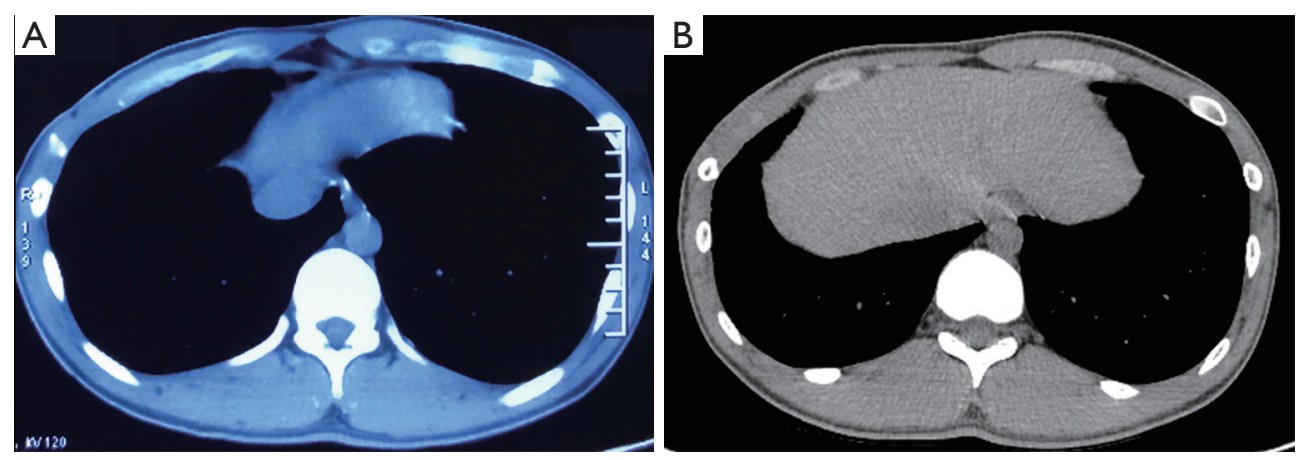

Figure 5 Chest CT showed proper healing the 3rd month (A) and the 6th month (B) after surgery.

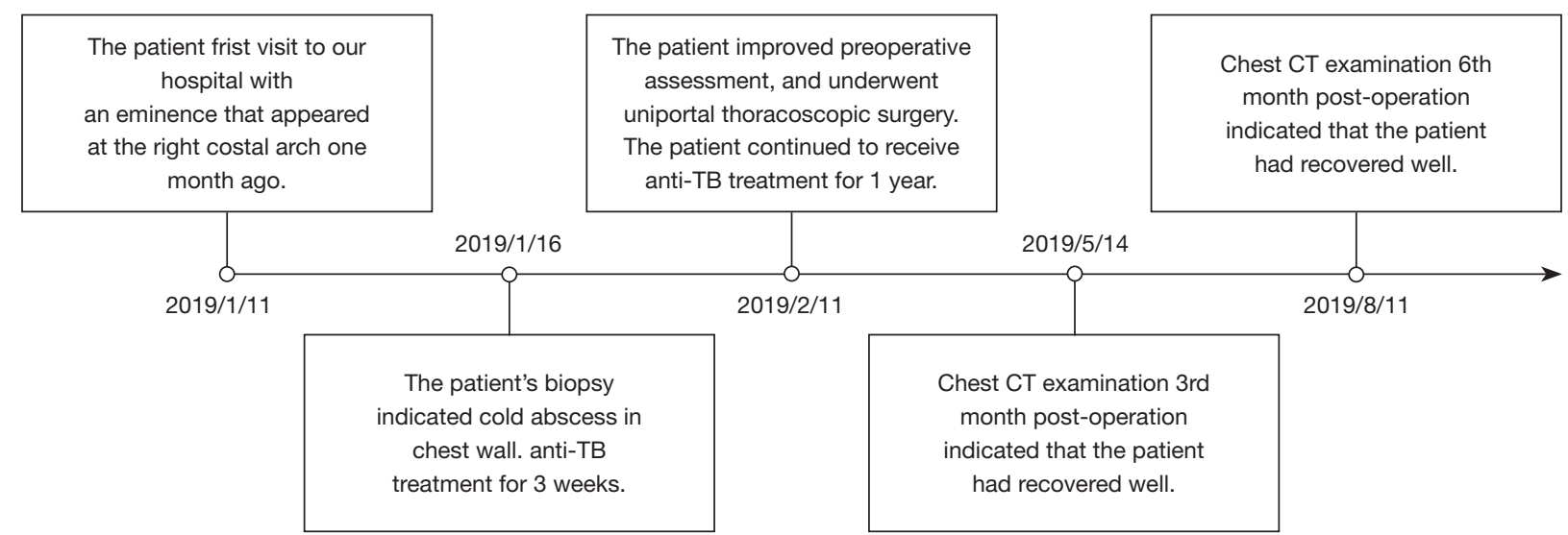

Figure 6 Timeline of diagnosis, treatment and prognosis in this case.

The hemostasis is stopped with dry gauze; after the gauze is removed, electrocoagulation is performed on the remaining bleeding sites to stop bleeding. Thoroughly remove the tuberculous lesions in the sinus tract and track the open blind ends. For patients with rib periosteum defect, bone destruction, and/or sinus tract or dumbbell-like abscesses on the deep side of the ribs, a wire saw is an ideal tool for removing the rib segment during uniportal-VATS. If the sinus tract is undermining multiple coastal beds, explore the direction of the sinus tract first. Remove the rib segments covering it; then use the curette or curved forceps to lift the outer wall of the sinus to avoid the injury of intercostal vessels and nerves. Cut open the coastal bed and its lower sinus wall along the directions of parallel intercostal vessels and nerves. Cut open and shorten the sinus tract beginning from the coastal bed next to the sinus ostium and remove the diseased tissues one by one.

\section{Dr. Yilei Zbang}

There were some limitations and disadvantages of VATS applications in thoracic surgery for a tuberculous collarbutton abscess of the chest wall involving ribs: (I) all surgical instruments are operated through one operation incision, and the operation process is certainly not as convenient as open surgery. (II) if there were many sinus tracts of abscess. The surgeons also should explore all the sinus tracts and eliminate them one by one. Sometimes open surgery is more intuitive than VATS.

There are some statuses unsuitable for VATS as follow: (I) the damaged ribs (and even the normal supporting ribs above the abscess) were difficult to be thoroughly resected; (II) the residual cavities were too large to be eliminated by VATS; (III) the residual cavities were large and needed muscular flaps with abundant blood supply to fill the cavities.

During open surgery, the damaged ribs (and even the normal supporting ribs above the abscess) must be 
thoroughly resected so that the soft tissues of the chest wall can entirely collapse to eliminate the residual cavity. If the residual cavity is large, a muscular flap with abundant blood supply can fill the cavity, with its bottom fixed with absorbable sutures and its peripheral areas fixed with silk sutures. For a large wound with massive exudate, a porous silicone tube can be placed for negative-pressure drainage, and the drainage tube is undermined into normal muscle tissue. If the patient has difficulty transferring a muscular flap or has multiple skin defects that may cause a primary closure to be impossible, the lesion is cleared, iodoform gauze may fill the cavity after the lesion is cleared away, and the dressing can be changed one after another.

\section{Can a minimally invasive surgery altogether remove abscesses and damaged ribs?}

\section{Dr. Zhiyong Su}

In recent years, VATS has increasingly been applied in the surgical treatment of lesions in the lungs, mediastinum, and esophagus; however, no literature has described its application in treating the dumbbell-shaped TB abscesses inside and outside the rib cage. The significant challenges include abscess drainage and resection of rib lesions. Our surgical design fully reflects the critical considerations in open surgery. Using the ultrasound and CT findings of the pus cavities inside and outside the rib cage, the working port was created at the lowest point of the pus cavity outside the chest wall. It has the following advantages: on the gap formed by the pus cavity, a surgical tunnel was established by using an incision protector, which enabled the removal of pus cavities inside and outside the rib cage via axial operation without a blind zone under the thoracoscope and facilitated the placement of the drainage tube at the lowest point after the operation. How to remove the damaged ribs in such a small space is another key to successful surgery. Under the guidance of a thoracoscope, the damaged ribs were pelted off with a periosteal stripping device and removed with a flexible wire saw. Using a wire saw, the creation of a surgical tunnel and the traction of the incision are vital steps in the surgery. Since there is no large incision (which is unavoidable in a traditional surgery), the working port can act as a drainage hole for placing a drainage tube. After surgery, compression bandaging will close the dead space, which solves the problem of the unhealed incision. Thus, no muscle flap is required for filling, which avoids more substantial trauma or scar, causing the chest wall more physically aesthetic after the surgery. Even if there is a recurrence, it is much easier for the operator to re-design an incision, which, however, might be challenging when the dehiscence of incision occurs after a traditional surgery using a large incision.

\section{Inputs from Head and Neck Surgeon Dr. Baiban $\mathrm{Su}$}

Incision protection sleeves are widely used in surgeries. During cervical tuberculous lymphadenopathy, we use them to protect the incision. By pulling them, we can create a potential tunnel gap to facilitate abscess removal. Thoracic surgeons place incision protection sleeves in the space between the chest muscles and the ribs to form a potential operating plane. By stretching the sleeves, they can create a simple trumpet-shaped device for flushing or pouring disinfectant into the abscess cavity. When the protective sleeves are rotated and tightened, they can temporarily seal the abscess cavity to prevent the overflow of pus or cleaning and disinfection solutions, and meanwhile, facilitate the operation via the camera port.

Several issues on this case were further discussed as follows.

\section{Question 1: Would you choose a minimally invasive surgery? Why?}

\section{Expert opinion 1: Dr. Michel Gonzalez}

The goal of surgery is to remove all infectious material, necrotic tissue (ribs, cartilage or lung) and prevent infection recurrence. The choice of a surgical approach for chest wall tuberculosis should be individualized and based on various criteria: (I) the presence of a sinus tract; (II) the size of the abscess; (III) the number of damaged ribs and (IV) the presence of concomitant pulmonary or mediastinal infection. Minimally invasive approaches allow a reduction of the surgical trauma and yields optimal aesthetic results in comparison with standard open approaches. I would choose a minimally invasive approach to manage a small lesion with 1 or 2 damaged ribs and if any pulmonary lesions are localized, as is the case in this report. This approach allows pus evacuation, damaged ribs removal and complete necrotic tissue debridement without major difficulties. In addition, in case of associated pulmonary infection, it would have been possible to remove parts of the lung parenchyma by wedge resection or debride an empyema. However, in presence of larger residual cavities, of extensive muscular debridement or of a sinus tract, I would recommend a standard incision in the midpoint of the lesion. In such cases, the application of a Vacuum-assisted closure device after extensive debridement can be useful to favor the healing of the debrided cavity. 


\section{Expert opinion 2: Dr. Marco Sperandeo}

The possibility of performing minimally invasive surgery is always desirable and, nowadays, this procedure is facilitated by the advance in surgical techniques and devices. Videoassisted thoracic surgery (VATS) has several advantages, including a smaller incision, no muscle damage and no dead space issue, which can lead to complication. In addition, it allows a shorter hospital stay and a more rapid recovery to daily life. In particular, one-port methods provide a more anatomic and direct view of the target tissue and allow bimanual instrumentation, thus avoiding the creation of torsional or dihedral angles by the instruments that is a limit in conventional multiportal VATS. As a result, the operation may be the same as an open procedure, with comparable results. Starting from these considerations, the choice of a uniportal approach seems to be the best solution in treating a tuberculous collar-button abscesses of the chest wall in an adult patient, as in the case report presented by the authors. This approach, in fact, has the further advantage of resulting in a single scar (instead of three) with less post-operative pain and stress for the patient, shorter hospital stays, and shorter convalescence.

\section{Expert opinion 3: Dr. Kyoji Hirai}

The point is that as described in manuscript an unmarried young patient strongly hoped the cosmetic surgery. Uncertain operation must be avoided. Whether the sinus tract communicating with abscess cavity and infected chest wall including the ribs can be treated correctly during U-VATS procedure or not is a key for the treatment of this disease. Generally, U-VATS is possible to be an uncertain treatment in view of complete extirpation of infected chest wall. However, in this case, localized abscess cavity with only two infected ribs (the 7th and 8th ribs) and no findings of extended inflammatory change to lung tissue may motivate the surgeon to perform U-VATS. Honestly, only limited patient with chest wall tuberculosis will be applied to U-VATS. Careful indication of U-VATS for chest wall tuberculosis should be considered and the accumulation of outcomes of this operation is required.

\section{Question 2: In your opinion, should surgery for chest wall tuberculosis be performed after systemic anti-tuberculosis treatment?}

\section{Expert opinion 1: Dr. Michel Gonzalez}

The rationale behind pre-operative anti-tuberculosis treatment is to reduce the number of tuberculosis bacteria during surgical procedure to reduce the risk of systemic $\mathrm{Tb}$ dissemination during surgery. However, there is no clear consensus regarding the optimal timing of preoperative anti-tuberculosis treatment, nor is there a widely agreed treatment plan for shorter-than-usual antibiotic treatments. Historically, it has been suggested that patients presenting chest wall tuberculosis should receive at least 2 months of pre-operative anti-tuberculosis treatment, but this approach should be balanced with a potential increased risk of drug resistance and poor compliance of the patient to the treatment. Recently, Zhang et al. have reported a $3.8 \%$ recurrence rate after ultra-short-course anti-tuberculosis pre-operative treatments (mean duration: $17 \pm 12.5$ days) in 263 patients with chest wall tuberculosis. This aggressive approach should be recommended to potentially reduce the length of hospital stay and to increase treatment compliance whilst minimizing issues of drug resistance. This is especially valid since these authors report that the post-operative recurrence rate is no different than after the usual, full-duration pre-operative anti-TB treatment (9).

\section{Expert opinion 2: Dr. Marco Sperandeo}

Musculoskeletal tuberculosis (TB) is a rare form of extrapulmonary TB that occurs in $1-3 \%$ of patients with TB. Among them, chest wall TB constitutes $1 \%$ to $5 \%$ of all cases of musculoskeletal TB. That's why there is no clear consensus regarding the optimal treatment modality for such condition. Anyhow, a medical treatment with anti-TB drugs is the first line therapy. The World Health Organization (WHO) and the British Thoracic Society recommend a treatment duration of 6 month for both extrapulmonary and pulmonary TB. Other institutions recommend 9 months of therapy for osteoarticular TB (ATS/CDC/IDSA). There are some series (10) reporting good results with only anti-tuberculosis drugs. But in other series (11), abscesses were not cured and even recurred or progressed despite adequate medical treatment. Indeed, it may be difficult for the systemic anti-TB drugs to reach the effective bactericidal concentration required in the abscess. Hence medical treatment alone may be not sufficient, some authors recommend combining medical and surgical management. In a study including 89 patients with chest wall abscess due to TB (12), recommended preoperative and post-operative anti-TB drugs and complete resection of chest wall mass including any suspicious ribs. This seems the best intervention protocol in order to avoid recurrences. In the case of TB abscesses, in the phase preceding the 
invasive surgery it is always better to carry out medical treatment (as the authors of the case report correctly did) in order to avoid the spread of the infection nearby. Furthermore, anti-TB treatment following surgery, under close laboratory monitoring, is also always advisable. The latter is appropriate in order to eliminate any micro foci of $\mathrm{TB}$ present in the body, not detectable by any diagnostic method.

\section{Expert opinion 3: Dr. Kyoji Hirai}

There are some cases that only use of anti-TB agents chest wall tuberculosis was fully healed, however, its use in long term leads to strong drug induced side effect. In this case, anti-TB agents were given for three weeks before operation, however there is no evidence that premedication is effective for TB collar-button abscess till now. Considering the U-VATS treatment for this disease, the possibility of incomplete extirpation is expected. The use of pre- and post-anti-TB agents is necessary to prevent the recurrence of chest wall tuberculosis.

If the surgeon does U-VATS with a planned operation, pre- and post-anti-TB treatment should be administered to reduce the occurrence of recurrence. However, in case that pre-anti-TB treatment will be significantly effective, careful indication and timing of operation must be determined.

\section{Question 3: Do you have any suggestions to prevent the recurrence of chest wall tuberculosis and reoperation?}

\section{Expert opinion 1: Dr. Michel Gonzalez}

The surgical principle of chest wall tuberculosis is to eliminate TB foci, to debride all necrotic tissue and to fill the residual cavity with healthy tissue. All damaged ribs should be resected to reduce the occurrence of residual disease. In case of doubt, a vacuum-assisted closure device can be placed temporarily and iterative dressings performed until granulation tissue is sufficiently formed. For patients with small abscesses, residual cavity formation can be avoided by simple pressure bandaging. However, in case of larger defects, debridement of surrounding tissue should be carried out until well vascularized tissue is reached. Use of non-resorbable mesh or thread should be avoided and muscular flap favored to fill any large residual cavity or defect. In the post-operative phase, anti-tuberculosis drug treatment should obviously be continued according to the drug sensitivity of the $\mathrm{Tb}$ bacteria strain. Regular liver and kidney function should be controlled as well as nutritional status to optimize wound healing.

\section{Expert opinion 2: Dr. Marco Sperandeo}

A TB chest wall abscess may results from a direct diffusion by the contiguous pleura and lung parenchyma, from the hematogenous spread by some underlying pathology or from infected lymph nodes through lymphatic route. As previously said, the anti-TB treatment following surgery is always advisable because it allows to sterilize any possible micro focus of bacilli eventually present in the body. In addition, a close diagnostic imaging and laboratory monitoring after surgery for over 1 year (with ultrasound and, eventually, MRI) could allow for early detection of reinfection and early medical treatment, avoiding the risk of any relapses and avoiding further interventions. Moreover, the epidemiological prevention of the patient (i.e., possible contacts with TB carriers) could be desirable, especially in the case of immunosuppressed patients.

\section{Expert opinion 3: Dr. Kyoji Hirai}

Needless to say, complete resection of abscess and infected ribs is linked with the prevention of recurrence of chest wall tuberculosis. Before operation, the assessment of bone lesions by bone scintigram and understanding of abscess spread by coronal and sagittal section by chest CT are useful in correct treatment of this disease might have been done. In this case, the size of abscess cavity was $10 \mathrm{~cm} \times 10 \mathrm{~cm} \times 3 \mathrm{~cm}$ and two ribs were extirpated by about $3 \mathrm{~cm}$. Though muscle flap plasty is difficult to make in U-VATS, insertion of muscle filling flap to dead space after treatment of abscess cavity may be effective for the prevention of the recurrence. Further devise in U-VATS may be necessary in the future. In several reports, postoperative administration of anti-TB agents for six to twelve months is appropriate and essential.

\section{Conclusions}

The key to the successful surgical treatment of chest wall tuberculosis is to eliminate the abscess cavity, remove the damaged ribs, reduce chest wall deformities, and prevent a postoperative recurrence, which can be achieved by the minimally invasive thoracoscopic surgery as it has no blind areas and allows the simultaneous removal of bone lesions. However, VATS surgery requires experience in open surgery and has a long learning curve. Also, the effectiveness and safety of such surgeries need to be further confirmed in studies with large sample sizes and long follow-up. Finally, the indications for these surgeries warrant further 
optimization.

\section{Acknowledgments}

Funding: None.

\section{Footnote}

Reporting Checklist: The authors have completed the CARE reporting checklist. Available at http://dx.doi.org/10.21037/ jtd-20-3504

Conflicts of Interest: All authors have completed the ICMJE uniform disclosure form (available at http://dx.doi. org/10.21037/jtd-20-3504). KH serves as an unpaid editorial board member of fournal of Thoracic Disease from Sep 2020 to Aug 2022. MG serves as an unpaid editorial board member of Fournal of Thoracic Disease from Dec 2018 to Nov 2020. The other authors have no conflicts of interest to declare.

Ethical Statement: The authors are accountable for all aspects of the work in ensuring that questions related to the accuracy or integrity of any part of the work are appropriately investigated and resolved. All procedures performed in studies involving human participants were in accordance with the ethical standards of the institutional and/or national research committee(s) and with the Helsinki Declaration (as revised in 2013). The Ethics Committee approved this study of the Affiliated Hospital of Chifeng University (No. FSYY202002). Written informed consent was obtained from all patients.

Open Access Statement: This is an Open Access article distributed in accordance with the Creative Commons Attribution-NonCommercial-NoDerivs 4.0 International License (CC BY-NC-ND 4.0), which permits the noncommercial replication and distribution of the article with the strict proviso that no changes or edits are made and the original work is properly cited (including links to both the formal publication through the relevant DOI and the license).
See: https://creativecommons.org/licenses/by-nc-nd/4.0/.

\section{References}

1. Bergeron EJ, Meguid RA, Mitchell JD. Chronic Infections of the Chest Wall. Thorac Surg Clin 2017;27:87-97.

2. Faure E, Souilamas R, Riquet M, et al. Cold abscess of the chest wall: a surgical entity? Ann Thorac Surg 1998;66:1174-8.

3. Sakuraba M, Sagara Y, Komatsu H. Surgical treatment of tuberculous abscess in the chest wall. Ann Thorac Surg 2005;79:964-7.

4. Lim SY, Pyon JK, Mun GH, et al. Reconstructive surgical treatment of tuberculosis abscess in the chest wall. Ann Plast Surg 2010;64:302-6.

5. Hakakian CS, Lockhart RA, Kulber DA, et al. Lateral Intercostal Artery Perforator Flap in Breast Reconstruction: A Simplified Pedicle Permits an Expanded Role. Ann Plast Surg 2016;76 Suppl 3:S184-S190.

6. Cho S, Lee EB. Surgical resection of chest wall tuberculosis. Thorac Cardiovasc Surg 2009;57:480-3.

7. Iseman MD. Extrapulmonary tuberculosis in adults. In: Iseman MD, editor. Clinician's Guide to Tuberculosis. Philadelphia: Lippincott, 2000:145-97.

8. Kim WJ, Kim WS, Kim HK, et al. Reconstruction of small chest wall defects caused by tubercular abscesses using two different flaps. Ann Thorac Surg 2018;106:e249-e251.

9. Zhang W, Chen J, Wu X, et al. Preoperative ultra-shortcourse chemotherapy combined with surgery for the treatment of chest wall tuberculosis. Infect Drug Resist 2020;13:2277-84.

10. Chen CH, Shih JF, Wang LS, et al. Tuberculous subcutaneous abscess: an analysis of seven cases. Tuber Lung Dis 1996;77:184-7.

11. Hsu HS, Wang LS, Wu YC, et al. Management of primary chest wall tuberculosis. Scand J Thorac Cardiovasc Surg 1995;29:119-23.

12. Paik HC, Chung KY, Kang JH, et al. Surgical treatment of tuberculous cold abscess of the chest wall. Yonsei Med J 2002;43:309-14.
Cite this article as: Bian $\mathrm{H}, \mathrm{Su} \mathrm{B}, \mathrm{Wu} \mathrm{C}$, Jiang T, Zhao X, Liu F, Lin K, Gao Q, Zhang Y, Gonzalez M, Sperandeo M, Hirai K, Su Z. Uniportal video-assisted thoracic surgery for a tuberculous collar-button abscess of the chest wall involving ribs: a case report. J Thorac Dis 2021;13(2):1291-1299. doi: 10.21037/ jtd-20-3504 